\title{
Congenital Curved Nail of the Fourth Toe with Subungual Hyperkeratosis
}

\author{
Kazuya Kashiyamaa, b Katsumi Tanaka ${ }^{a}$ \\ aDepartment of Plastic and Reconstructive Surgery, Nagasaki University Hospital, Nagasaki, Japan; \\ ${ }^{b}$ Department of Plastic Surgery, Maxillofacial Surgery, Aesthetic Surgery, Yuuai Medical Center, Okinawa, Japan
}

\section{Established Facts}

- Congenital curved nail of the fourth toe (CNFT) is a rare nail deformity.

- Distal bone hypoplasia can develop.

\section{Novel Insights}

- A novel surgical technique was employed to treat CNFT with thickening and hyperkeratosis at the hyponychium and peripheral nail bed.

\section{Keywords}

Congenital · Curved nail · Fourth toe

\section{Abstract}

Congenital curved nail of the fourth toe (CNFT) is a rare nail deformity. We report a case of CNFT with thickening and hyperkeratosis at the hyponychium and peripheral nail bed that was successfully treated using a novel surgical technique. A 7-year-old Japanese boy had a clawed nail with thickening and hyperkeratosis at the hyponychium and peripheral nail bed on his bilateral fourth toes. His chief complaint was pain. The nails of the bilateral fourth toes curved plantarly and exhibited thickening and hyperkeratosis of the hyponychium and peripheral nail bed. A surgical approach was planned to improve the condition. The distal phalanges that deviated and formed a point toward the nail bed were partially shaved, and a soft tissue flap was created in the fin- ger pad and shifted to cover the distal phalanges. The dorsal side of the distal phalanges was covered by the nail bed flap. Recurrence has not been observed for 3 years after surgery. There is limited evidence supporting surgical approaches for CNFT. This approach may be an effective treatment option for CNFT with thickening of the nail bed.

(c) 2021 S. Karger AG, Basel

\section{Introduction}

Congenital curved nail of the fourth toe (CNFT) is a rare nail deformity, and it is rarely associated with other genetic anomalies [1]. Only a few cases have been reported, with limited description of surgical approaches [2]. We report a case of CNFT with thickening and hyperkeratosis at the hyponychium and peripheral nail bed that was successfully treated by a novel surgical technique.

$\begin{aligned} & \text { karger@karger.com } \\ & \text { www.karger.com/sad }\end{aligned}$
Karger $\%$



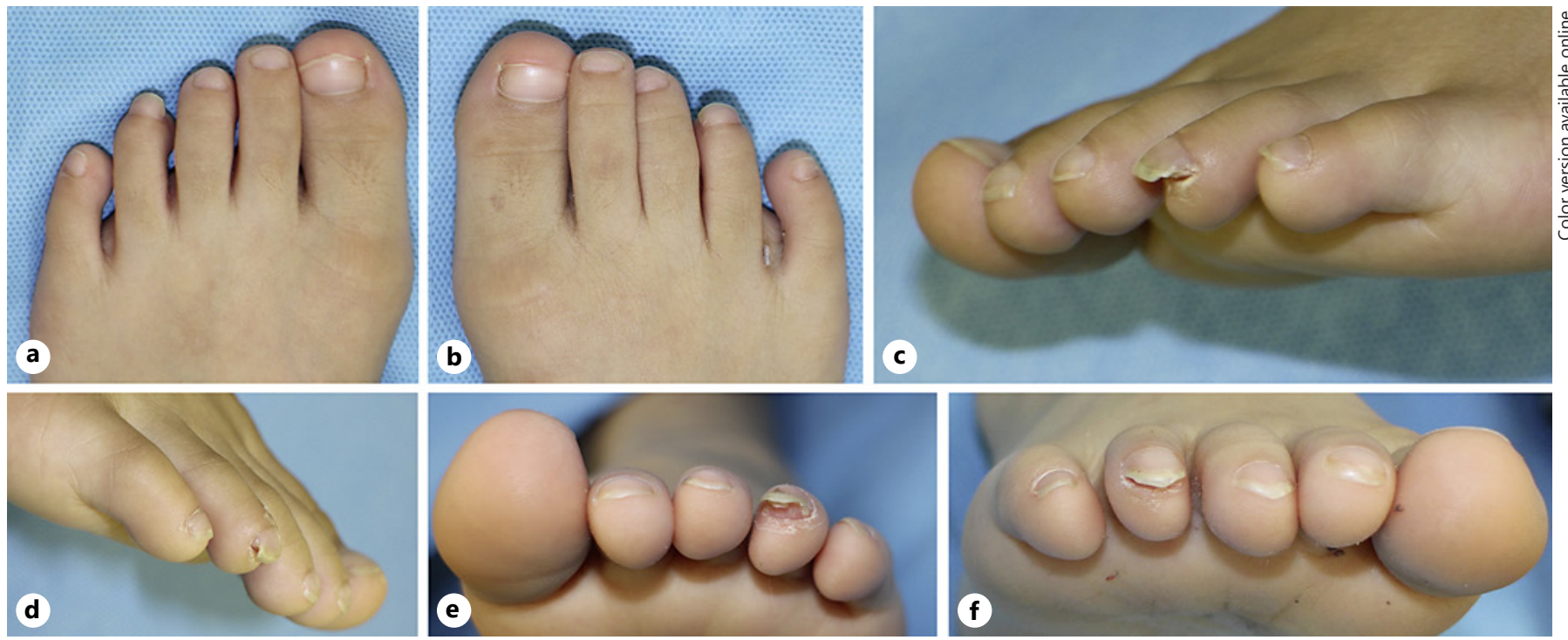

Fig. 1. Findings from the initial assessment. Shortening of the fourth toes was observed bilaterally. In addition, thickening and hyperkeratosis of both fourth toes were observed at the hyponychium and peripheral nail bed. a Dorsal view of the left foot. b Dorsal view of the right foot. c Lateral view of the left foot. $\mathbf{d}$ Lateral view of the right foot. e Frontal view of the left foot. f Frontal view of the right foot.
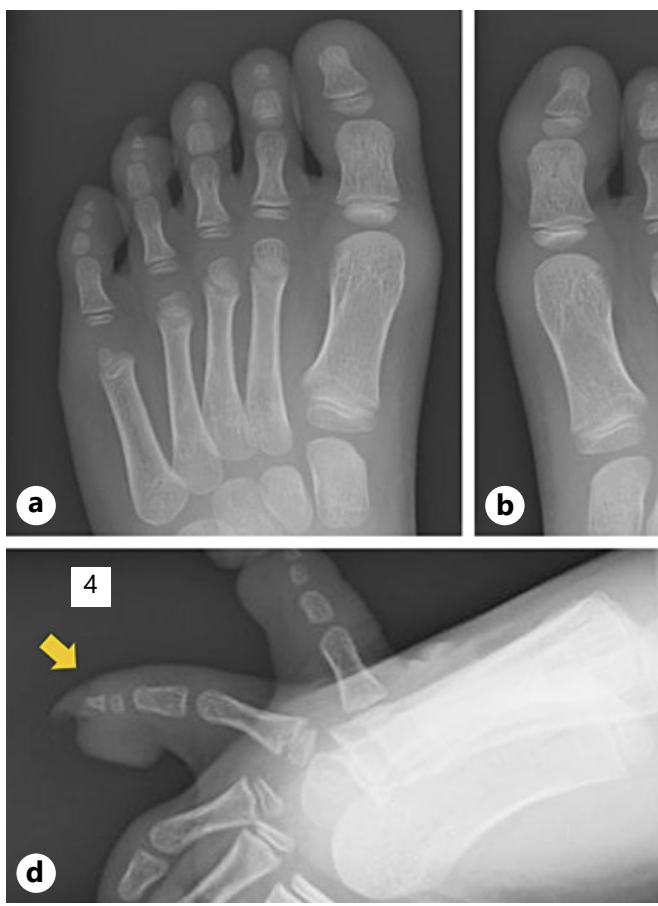
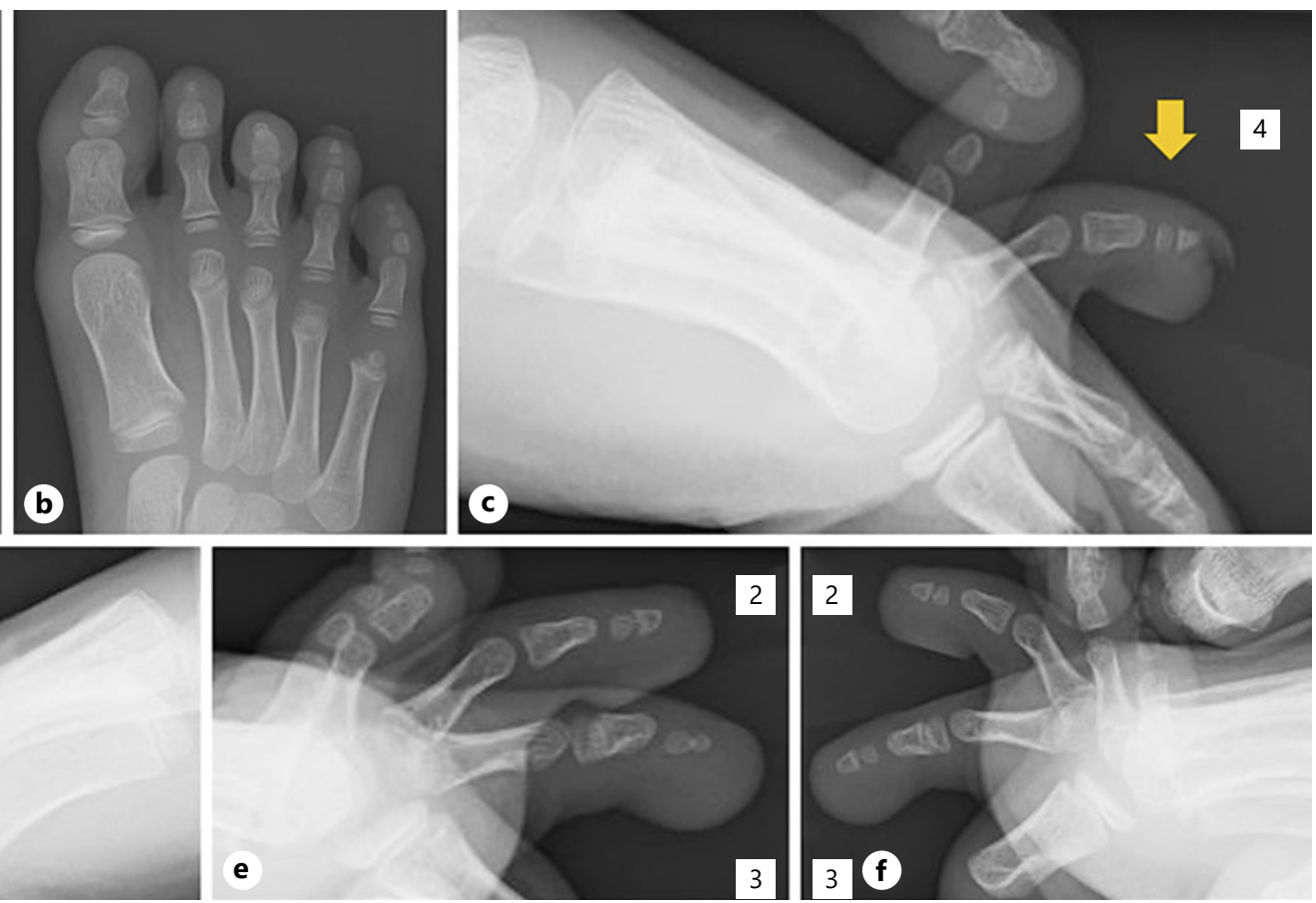

Fig. 2. Plain radiography at the time of the initial assessment. The distal phalanges of the fourth toes were slightly small and dorsally deviated. a Dorsal view of the left foot. $\mathbf{b}$ Dorsal view of the right foot. c Lateral view of the left fourth toe (arrow: fourth toe). d Lateral view of the right fourth toe (arrow: fourth toe). e Lateral view of the left second and third toes. $\mathbf{f}$ Lateral view of the right second and third toes. 


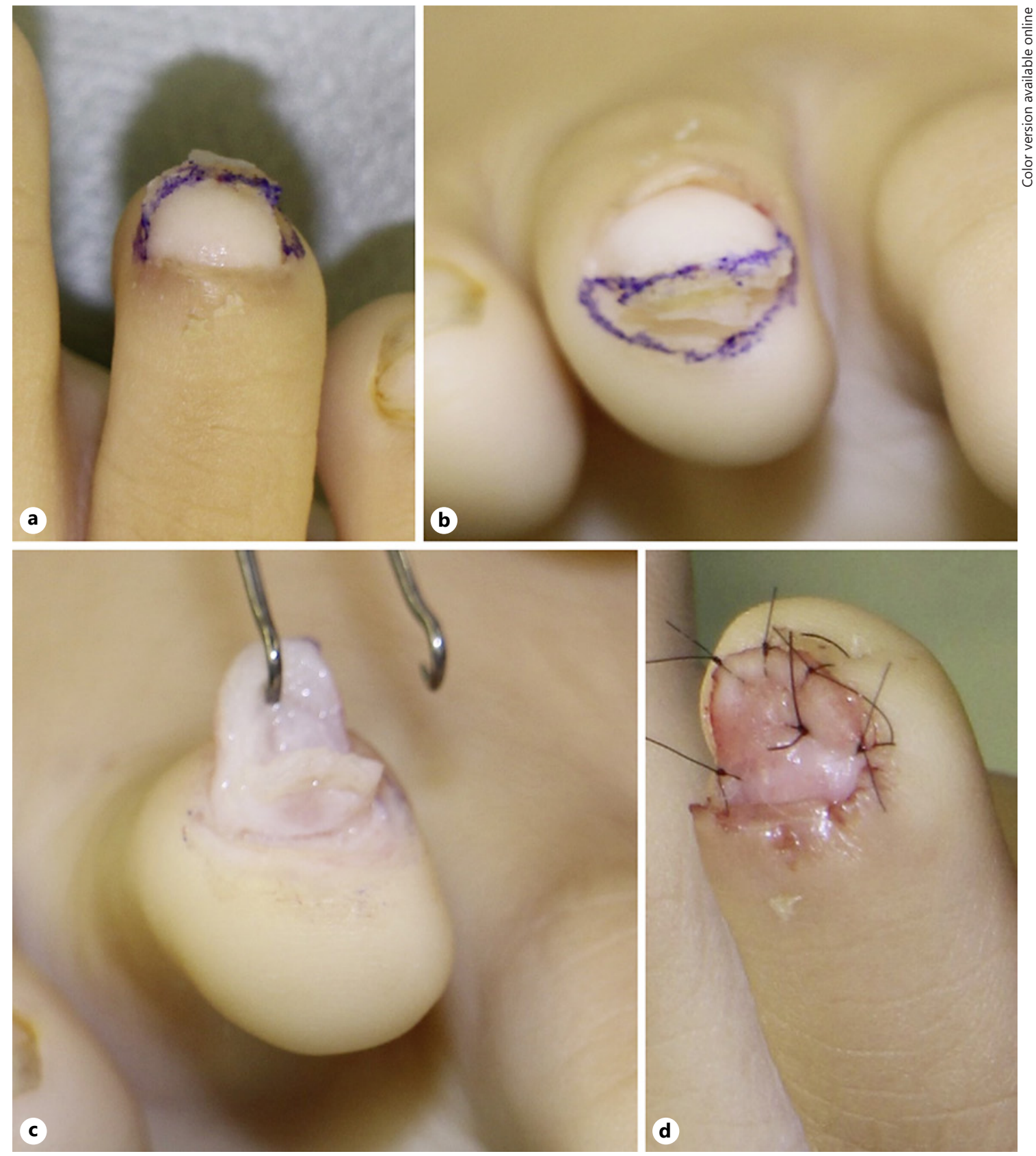

Fig. 3. Surgical findings (right fourth toe). a Skin incision (dorsal view). b Skin incision (frontal view). c A flap was lifted from the nail bed. Deviation of the distal phalanx was observed. $\mathbf{d}$ A flap from the finger pad was used to cover the distal phalanx. Thickened tissue of the nail bed was removed, and formation of the new nail bed was observed. The flap from the finger pad shifted to form finger tips.

\section{Patient and Methods}

\section{Case}

A 7-year-old Japanese boy had a clawed nail with thickening and hyperkeratosis at the hyponychium and peripheral nail bed on his bilateral fourth toes. His chief complaint was pain in his fourth toes that prevented him from playing football because his nails were catching on something. The nails of the bilateral fourth toes curved plantarly, and exhibited thickening and hyperkeratosis at the hyponychium and peripheral nail bed (Fig. 1). The other toenails and hand nails were normal. He had no family history or notable past history such as trauma. Preoperative X-ray re- 


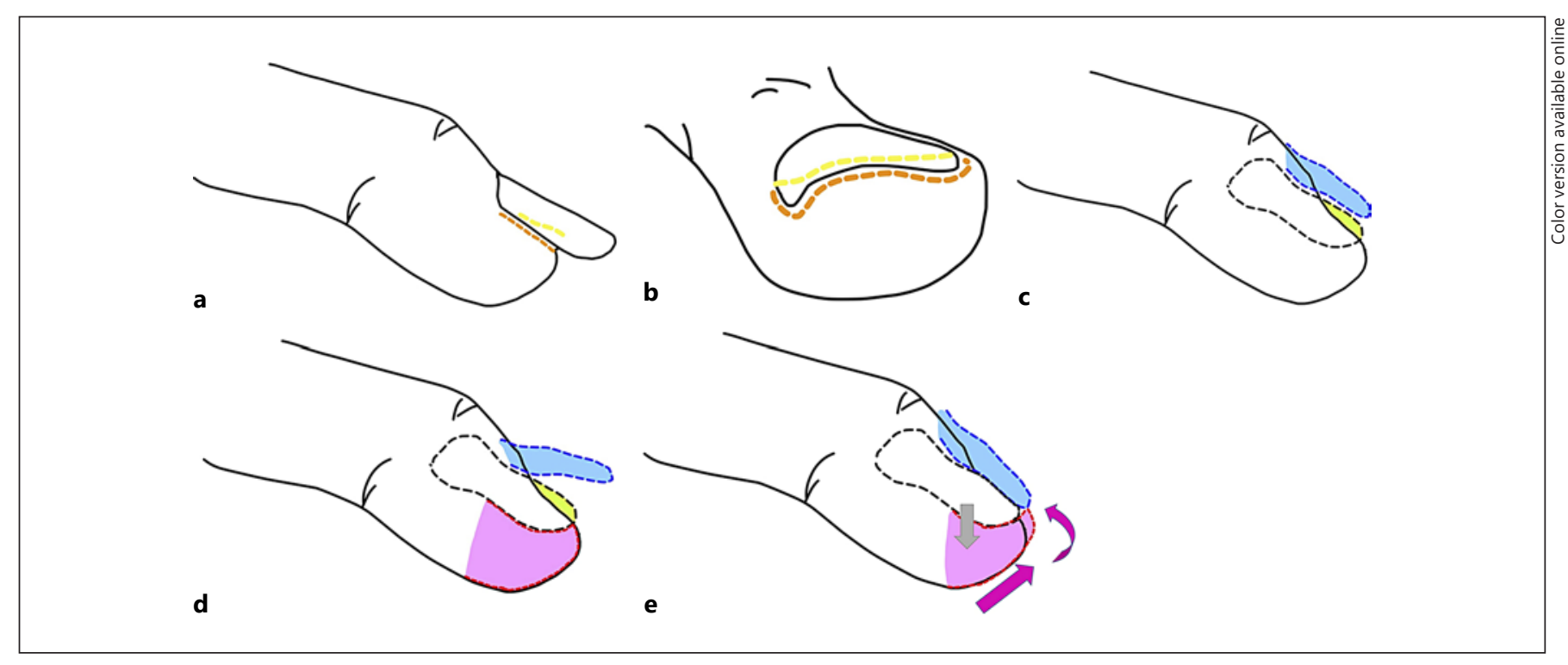

Fig. 4. Schematic of the surgical procedure. a Skin incision (lateral view). A skin incision was made at the border of the nail bed and skin. Orange: skin incision to lift a flap from the nail bed. Yellow: skin incision to resect the thickened nail bed. b Skin incision (frontal view). c The nails were removed before partially resecting the thickened nail bed. Blue: a flap from the nail bed that was lifted on the periosteum. Green: distal phalanx that deviated to deform the nail bed. d The distal phalanges were shaved partially (green). A soft tissue flap was lifted from the finger pad (red). e The flap from the finger pad was rotated to shift it forward and upward in order to cover the tip of the distal phalanges. The dorsal side of the phalanges was covered by the flap from the nail bed. vealed that the lengths of the left and right metatarsals were normal, but the distal phalanges of the fourth toes were slightly small and dorsally deviated (Fig. 2). In order to improve his condition, surgery was performed under general anesthesia. After removing the toenails, thickened tissue that built up on top of the nail bed was resected. The finger bed was unevenly deformed and formed a point as the distal phalanges shifted. A semicircular skin incision line was drawn on the skin between the skin and the finger bed (Fig. 3a, b, 4a, b). After creating the incision, the nail bed flap was elevated on the periosteum of the distal phalanges (Fig. 3c, $4 \mathrm{c}, \mathrm{d})$. Next, the distal phalanges that deviated toward the nail bed and were internally putting pressure on the nail bed were partially shaved using bone files and Luer Bone Rongeur forceps (Fig. 3c, 4d). Soft tissue was separated from a section between the distal phalanges and the finger pad to create a soft tissue flap from the finger pad. The flap from the finger pad was shifted forward and upward to cover the tip of the distal phalanges, and a monofilament absorbable suture was used to suture the flap with tissue around the distal phalanges (Fig. 4e). The dorsal side of the distal phalanges was covered by the nail bed flap, and the flap from the finger pad was sutured with the nail bed flap to close the surgical site (Fig. 3d, 4e). Recurrence of the thickening and hyperkeratosis at the hyponychium and peripheral nail bed has not been observed for 3 years after surgery (Fig. 5). The curved nail slightly remained, requiring nail and foot care.

\section{Discussion}

CNFT is a rare nail deformity. Iwasawa et al. [3] first reported a plantarly curved nail on the fourth toe as CNFT in 1991. Since then, a few cases have been reported [4-8]. Almost all reports were from Asian countries, such as Japan, Taiwan, and Korea, with only one case reported from Brazil [1]. In previous reports, there were more males than females ( 21 males and 5 females) and more bilateral cases than unilateral cases (22 and 5 patients, respectively). Eleven patients had a similar toe nail deformity in their family history. One patient had thumb polydactyly, and 3 had cleft lip and palate [1]. CNFT is characterized by a plantarly curved nail of the toe, which is parallel to the longitudinal axis of the distal phalanx. The cause of disease is unknown, but it is considered to be a mesodermal abnormality rather than an ectodermal abnormality $[2,9]$. CNFT was previously reported to be due to shortening of the distal phalanx on the bilateral or unilateral fourth toe with soft tissue hypoplasia of the toe tip $[2,5]$. As our patient was only 7 years old at the time of surgery, the distal phalanges were not fully mature. Thus, we were unable to confirm shortening or hypoplasia of the distal phalanges on en face $\mathrm{X}$-ray images. However, slight hypoplasia was observed on the lateral view 


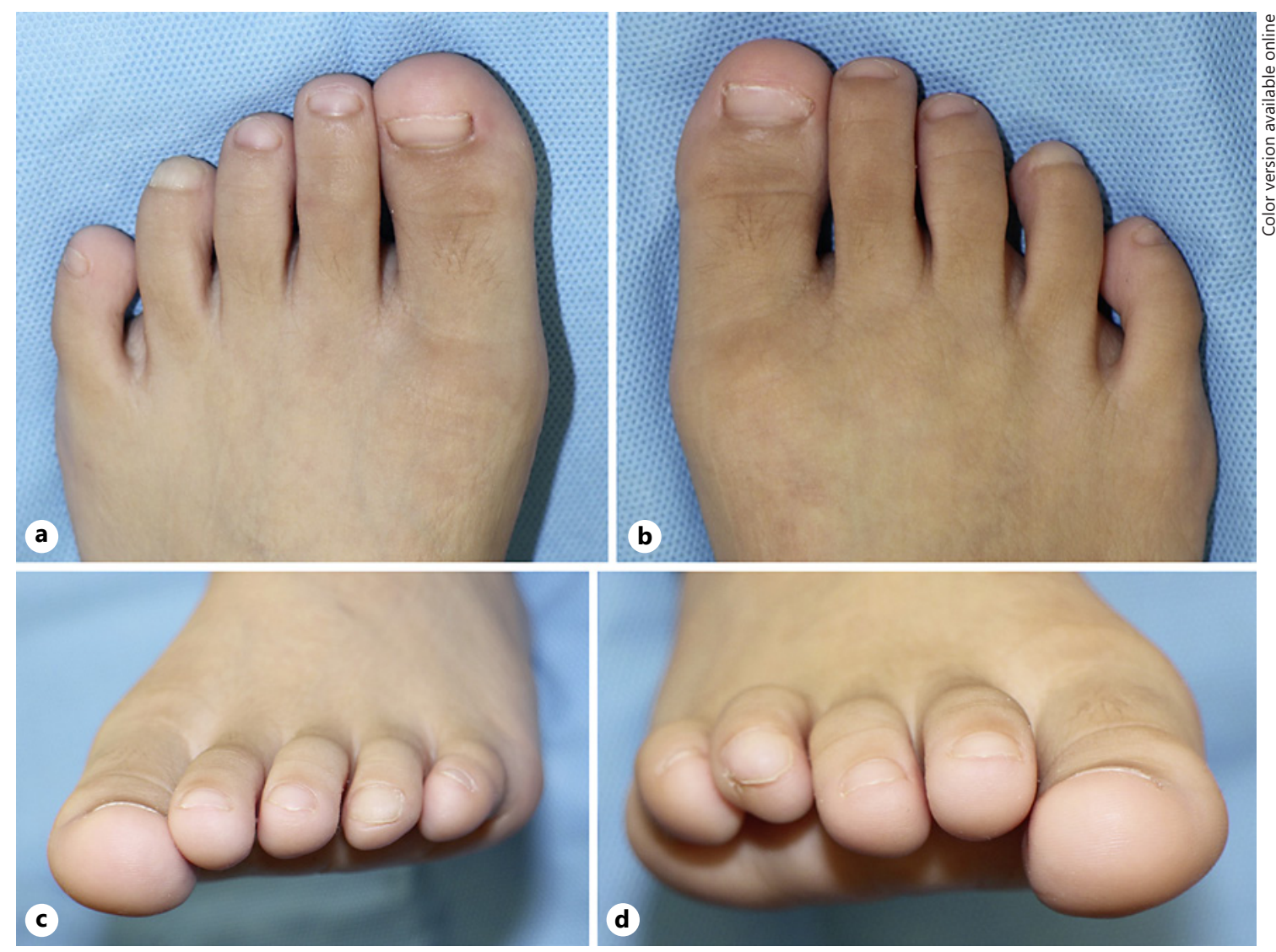

Fig. 5. Recurrence of the thickening of the nail bed has not been observed for 3 years after the surgery. a Left foot (dorsal view). b Right foot (dorsal view). c Left foot (frontal view). d Right foot (frontal view).

when compared with the distal phalanges of the other toe nails. The claw-shaped nail deformity was accompanied by bone and soft tissue deformity as secondary symptoms. In our case, subungual thickening and hyperkeratosis were tertiary developments. These phenomena may have resulted from the pressure that was applied internally on the nail bed by the distal phalanges that deviated dorsally and from repeated pressure that was applied dorsally by catching the nail-free edge when putting on shoes or socks.

The association with other congenital anomalies, such as cleft lip, alveolus, and plate, has been reported. However, almost all patients had no other abnormalities [1]. Our patient had no other congenital abnormalities. The treatments for CNFT are roughly divided into 2 methods. One is daily nail care, and the other is surgical treatment. Mitsukawa et al. [2] reported surgical treatment for CNFT using a V-Y advancement flap from the pad of the toe to reconstruct the tip of the toe and nail bed. Hard tissue reconstruction is not recommended for patients in the growth stage, and bone extension is difficult because the distal phalanx of the toe is small. As such, we developed a novel technique to cover the bone spur in the distal phalanges using flaps from the nail bed and finger pad. This procedure is relatively simple and may be less painful for patients because it does not leave much scar tissue. We left the periosteum in order to prevent growth defects and ensured that a sufficient amount of soft tissue was placed on the side of the bone as we lifted the flap from the finger pad in order to maintain blood flow to the bone. Recurrence of the thickening and hyperkeratosis of the hyponychium and peripheral nail bed has not been observed for 3 years after surgery. To date, the patient has no growth defects in his toes. The curved nail slightly remained, requiring continuous nail and foot care.

Unlike lesions of the hands, CNFT is a lesion of the toes and often goes unnoticed by patients unless symptoms, such as pain, develop. Thus, patients often do not visit clinics for CNFT. The high incidence of CNFT in 
Japan, Taiwan, and Korea may thus be a reflection of the cultural habits as individuals in these countries often remove their shoes at home. The paucity of reports on CNFT also suggests that there is little awareness of this disease in the medical community. Patients who present with deformity of the nails and distal segments may be misdiagnosed with brachydactyly or nail bed abnormalities. Thus, the incidence of CNFT is likely underreported. CNFT should be considered as one of the conditions that causes defects in the nail bed of toe nails.

\section{Conclusion}

CNFT is a rare congenital nail disorder. Our novel surgical approach was effective for treating CNFT with thickening of the nail bed. This approach may be selected as one of the options for CNFT with thickening of the nail bed.

\section{Statement of Ethics}

The study was performed in accordance with the principles of the Declaration of Helsinki. Written informed consent was obtained from the patient and their family for publication of this case report and any accompanying images.

\section{Conflict of Interest Statement}

No grant support from public institutions or private enterprises was received for this case report.

\section{Funding Sources}

No grant support from public institutions or private enterprises was received for this case report.

\section{Author Contributions}

K.K. conceived and wrote the article. K.K. and K.T. were involved in treating the patient. K.K. participated in editing the manuscript critically. All authors declare that they contributed to this article and that they have read and approved the final manuscript.

\section{References}

1 Di Chiacchio N, Noriega L, Di Chiacchio NG, Ocampo-Garza J, Padoveze EH. Congenital curved nail of the fourth toe associated with oral disorders. Skin Appendage Disord. 2017; 3(4):182-5.

2 Mitsukawa N, Karube D, Saiga A, Akita S, Kubota Y, Kuriyama M, et al. Surgical treatment for congenital curved nail of the fourth toe. Ann Plast Surg. 2015;74(3):361-4.

3 Iwasawa M, Hirose T, Matsuo K. Congenital curved nail of the fourth toe. Plast Reconstr Surg. 1991;87(3):553-4.
4 Yotsumoto S, Kanzaki T. Curved nail of the fourth toe. J Am Acad Dermatol. 1999;40(1): 124-5.

5 Lin YC, Wu YH, Scher RK. Congenital curved nail of the fourth toe: three different clinical presentations. Pediatr Dermatol. 2007;24(4): 380-3.

6 Baba N, Kawai I, Kanekura T, Hamada Y. Congenital curved nail of the fourth toe: case report. Nshinihon Hifu. 2009;71(4):455.
7 Skurai E, Sato R, Watabe D, Mori S. Congenital curved nail of the fourth toe: case report. Hifurunsyo. 2016;70(4):353-6.

8 Morigami J, Morigami T, Kuvota Y. Congenital curved nail of the fourth toe: case report. Nihon Hifuka. 2017;127(9):2145-6.

9 Choi YH, Song CH, Lee JS, Lee SJ, Kim DW, Lee WJ. Congenital malalignment of the fourth toenail with congenital curved nail of the contralateral fourth toe. Ann Dermatol. 2013;25(1):133-4. 